\title{
Trastornos del habla en el síndrome X frágil. Una revisión bibliográfica
}

Miriam Campos Guzmán - Universitat de València

Diana Marín Suelves - Universitat de València

$\mathrm{M}^{\mathrm{a}}$ Inmaculada Fernández Andrés - Universitat de València
0000-0002-9877-3137

0000-0002-5346-8665

0000-0003-2808-0949

Recepción: 2.01.2019| Aceptado: 21.01.2019

Correspondencia a través de ORCID: Ma Inmaculada Fernández iD 0000-0003-2808-0949

Citar: Campos Guzman, M., Marin Suelves, D. y Fernandez Andres, MI. (2019). Trastornos del habla en el síndrome X frágil. Una revisión bibliográfica. ReiDoCrea, 8, 43-54. [ ]

Resumen: El síndrome $X$ frágil (SXF) es un trastorno genético causante de discapacidad intelectual hereditaria. Su prevalencia es mayor en los hombres. En este artículo se presenta una revisión bibliográfica para analizar por una parte su grado de afectación lingüística y por otra la efectividad de su evaluación o intervención. A partir de determinados criterios de inclusión y exclusión, se ha analizado 19 artículos cuyos resultados han mostrado una variabilidad dependiente de los instrumentos aplicados, siendo especialmente eficaces los basados en el lenguaje guiado. Por tanto, se podría extraer conclusiones favorables a la hora de establecer en un futuro programas de evaluación o intervención ajustados a las necesidades de la población SXF, con ciertas garantías de éxito.

Palabras clave: Síndrome X frágil | Trastornos del habla

Speech disorders in fragile $X$ syndrome. A bibliographical review

\begin{abstract}
Fragile X Syndrome (SXF) is a genetic disorder that causes hereditary intellectual disability. Its prevalence is higher in men. In this article, a bibliographical review is presented to analyse, on the one hand, the degree of linguistic affectation and, on the other, the effectiveness of its evaluation or intervention. Based on certain inclusion and exclusion criteria, 19 articles were analysed, the results of which show a variability dependent on the instruments applied, with those based on guided language being especially effective. Therefore, favourable conclusions can be drawn when it comes to establishing evaluation or intervention programs in the future that meet the needs of the SXF population, with certain guarantees of success.
\end{abstract}

Keywords: Fragile X Syndrome | Speech Disorders

\section{Introducción}

Es en 1943 cuando el médico James Martin y la genetista Julia Bell estudiaron las peculiaridades físicas del síndrome que denominaron Martin-Bell. Años después, Lubs (1969) estudió varios varones de una misma familia que presentaban retraso mental actualmente discapacidad intelectual (DI). A través de un estudio cromosómico de las aportaciones de dos de los hermanos y de su madre, se descubrió una alteración en el brazo distal del cromosoma X, denominado "sitio frágil" y describió el fenotipo físico del trastorno, uniendo así ambos estudios y estableciéndose bajo el mismo cuadro clínico y denominación como síndrome de X frágil (SXF). Sutherland (1977) confirmó que el "sitio frágil" se localizaba en la región Xq27.3, dependiente del ácido fólico además descubrió que el trastorno era significativamente mayor y el grado de discapacidad era más severa en varones que en mujeres. Verkerk et al. (1991) mediante el desarrollo de sondas moleculares próximas al "sitio frágil", estableció definitivamente la ubicación del gen causante en el FMR1 (fragile mental retardation-1) dando lugar a la falta de síntesis de la proteína FMRP, abundante en las neuronas, de ahí que la característica principal del síndrome sea la discapacidad intelectual.

Su desarrollo en el individuo depende del número de tripletes de las bases nitrogenadas CGG (Citosina-Guanina-Guanina) que contiene la secuencia de ADN. Se 
clasifica en dos estados posibles en base al número de repeticiones, oscilando entre 200-230 para considerarse una mutación completa en cuyo caso el gen está totalmente inactivo y no sintetiza la proteína o entre 61-200 para considerarse una premutación en cuyo caso el funcionamiento del gen no está tan afectado para que aparezcan síntomas de SXF. Dicho número de repeticiones puede aumentar al transmitirse de una generación a otra, pues el síndrome sigue un patrón de herencia dominante logado al cromosoma X. Como indica Fu et al. (1991) los portadores de mutación completa no se suelen reproducir.

Murray, Webb, Grimley, Conway y Jacobs (1998) descubrieron un sub-fenotipo basado en el fallo ovárico prematuro (FOP) en mujeres con pre-mutación, sugiriendo que el gen interviene en el desarrollo y maduración del ovario, causando menopausia precoz, generalmente antes de los 40 años, con deficiencia de hormonas sexuales. Hagerman et al. (2001) descubrió el segundo sub-fenotipo en adultos con pre-mutación, caracterizado por un temblor progresivo, ataxia, déficit cognitivo, atrofia cerebral e impotencia sexual que suele aparecer pasados los 50 años.

Actualmente se considera que el SXF es el segundo trastorno genético causante de discapacidad intelectual hereditaria, únicamente precedido por el síndrome Down (SD) y relacionado en gran medida con el trastorno del espectro autista (TEA). Según García, Rigau y Artigas (2006), los niños SXF tienden a presentar puntuaciones altas en los instrumentos diagnósticos de autismo, lo cual está determinado por el hecho de que comparten síntomas.

Respecto al fenotipo físico que lo caracteriza, Ribate, Pié y Ramos (2010) destacan la macrocefalia, las orejas grandes y separadas con hendidura superior del lóbulo, una cara alargada y estrecha, estrabismo, paladar ojival, mandíbula inferior saliente y dientes apelotonados, laxitud articular y pies planos. En la edad adulta se pueden desarrollar de manera excesiva los testículos, denominado como macroorquidismo.

Con relación al fenotipo conductual, según indican Brun y Artigas (2001) variará en función del género o estado que presentan los afectados y la edad o etapa evolutiva. Como ejemplo de la citada variabilidad individual, se manifiesta más leve en las mujeres mientras que en la etapa previa a la pubertad es más persistente. Son habituales ciertas características como son, en el ámbito motor según Robles (2011) el uso y abuso de movimientos estereotipados, sean aleteos, mordida de manos o llevarse a la boca los puños de los jerséis. En el ámbito cognitivo, según Ferrando, Banús y López (2003) la discapacidad intelectual la presentan la totalidad de los varones con mutación y el $70 \%$ de las mujeres. Según Brun y Artigas (2001) procesan la información de manera visual más que auditiva, por su mayor facilidad para reconocer o recordar los estímulos visuales que demandan menos atención. En el ámbito social, la impulsividad, impaciencia, hiperactividad y el déficit de atención son frecuentes en la mayoría, debido a las cuales les cuesta tolerar la frustración. De acuerdo con Artigas, Gabau y Guitart (2006) responden con ansiedad ante situaciones o personas desconocidas por su timidez y evitan el contacto ocular con su interlocutor, apareciendo en ocasiones el mutismo selectivo. Sin embargo, según Robles (2011) no son indiferentes ante la interacción social, incluso provocándola y la evitación de la mirada no se da siempre, sino que es selectiva, dirigida más a los rostros conocidos.

Dentro del fenotipo conductual, cabe destacar el ámbito lingüístico y las características que caracterizan al SXF, ya que se ha observado cierto retraso en el desarrollo de sus habilidades lingüísticas. De acuerdo con Brun y Artigas (2001) y García, Rigau y Artigas (2006), presentan trastornos del lenguaje en todos sus componentes: el fonológico, sintáctico, semántico, pragmático y en general, el comprensivo. Según 
Roberts, Mirrett y Burchinal (2001) el problema es mayor a nivel de expresión que de comprensión y según Rondal y Ling (2015) en el ámbito fonológico, morfosintáctico y pragmático (citado en Huelmo, Martínez y Diez, 2017, p. 69).

Respecto a la adquisición del lenguaje, de acuerdo con Brun y Artigas (2001) la aparición de las primeras palabras es tardía, generalmente hasta los 3 años o más, sin embargo, su comprensión es buena y una vez adquirido tienen cierta facilidad para ampliarlo con vocabulario nuevo, aunque suele ser repetitivo y con abuso de ecolalias.

En cuanto a los componentes fonético y fonológico, según Fürgang (2001) tienen dificultad en ordenar y secuenciar los sonidos, y por tanto en su producción, convirtiendo su lenguaje en palabras aisladas e ininteligible en ocasiones ya que les resulta difícil la emisión de palabras con un número elevado de sílabas. Sin embargo, tienen memoria auditiva a largo plazo y buena capacidad de imitación, favoreciéndoles la estructuración correcta de las frases en el discurso, aunque a veces su habla sea rápida o entrecortada o con volumen insuficiente para hacerse oír.

Finalmente, con relación al componente pragmático, hay que destacar que es el más débil y por tanto el menos desarrollado en el SXF, confirmado por Koover, McDuffie, Abbeduto y Brown (2012) o Haebig, Sterling y Hoover (2016). Según Brun y Artigas (2001) tienden a no respetar el turno de palabra y les cuesta mantener el tema de conversación, dándose frecuentemente el lenguaje tangencial, tampoco realizan inferencias previas, por lo que, entienden todo de manera literal, costándoles comprender los dobles sentidos o las frases hechas. Klusek, McGrath, Abbeduto y Roberts (2016) han confirmado que la dificultad pragmática en madres con SXF, influye en el desarrollo del lenguaje en sus hijos con el síndrome, posicionándolos en una situación más vulnerable debido a su predisposición genética.

En cuanto al tratamiento médico del síndrome, es sintomático y aborda los diferentes trastornos que presenta cada caso (citado en Ferrando, Banús y López, 2003, p. 140). Con respecto al tratamiento logopédico, Robles (2011) y Robles, Sánchez y Valencia (2013) han confirmado a través de sus investigaciones, la efectividad de los CADIT (Centros de Atención y Desarrollo Infantil Temprano). Éstos intervienen mediante programas de atención temprana las áreas cognitiva, lingüística y motora a niños entre los 0 y 6 años con el objetivo de favorecer su integración escolar y social, así como su autonomía personal, junto con el asesoramiento a las familias.

\section{Objetivo}

A partir de esta información, se considera relevante analizar las investigaciones recientes centradas en la evaluación o intervención del SXF en los diversos componentes lingüísticos, con el fin de establecer futuras pautas en base a la evidencia científica.

\section{Métodos}

En las siguientes líneas se presentan los criterios de inclusión y exclusión tras los cuales se seleccionó los artículos incluidos en la revisión bibliográfica.

Respecto a los criterios de inclusión fueron los siguientes:

- Artículos publicados en las bases de datos de reconocido prestigio Dialnet, Educational Resource Information Center (ERIC), Google Academy y Scopus. 
- Artículos científicos de investigación publicados en la red, centrados en el estudio del síndrome X Frágil.

- Artículos publicados entre el año 2000-2018 ya que es un rango de búsqueda efectivo para encontrar un número suficientemente amplio de artículos.

- Artículos dirigidos a la dimensión lingüística del trastorno, a partir de las palabras clave: trastornos en el habla, discapacidad intelectual, evaluación, intervención, alumnado. Buscadas también en inglés como: speech disorders, intellectual disability, evaluation, intervention, students.

- Artículos que acogen un rango de edad comprendido entre los 0 y los 18 años. Con excepciones minoritarias de grupos en edad adulta, pues se ha considerado también esta franja de edad como relevante pues el objetivo era valorar la evolución de su vocabulario o habla, así como la influencia de progenitores a hijos con el síndrome.

- Artículos redactados en castellano e inglés.

En cuanto a los criterios de exclusión fueron los siguientes:

- Artículos publicados en bases de datos de pago o de acceso restringido.

- Artículos que eran explicaciones teóricas del trastorno.

- Artículos dirigidos a la dimensión genética o a la edad adulta.

- Artículos incompletos o que se repetían en las diferentes bases de datos.

A partir de las palabras clave mencionadas anteriormente se realizó una búsqueda en las diversas bases de datos obteniendo un total de 28 artículos de los cuales, atendiendo a los criterios de inclusión y exclusión, se seleccionaron 19 y se desecharon los 9 restantes. Todo ello queda reflejado en la siguiente figura (1) que muestra el diagrama de flujo del método empleado para la búsqueda de los artículos de la revisión bibliográfica.

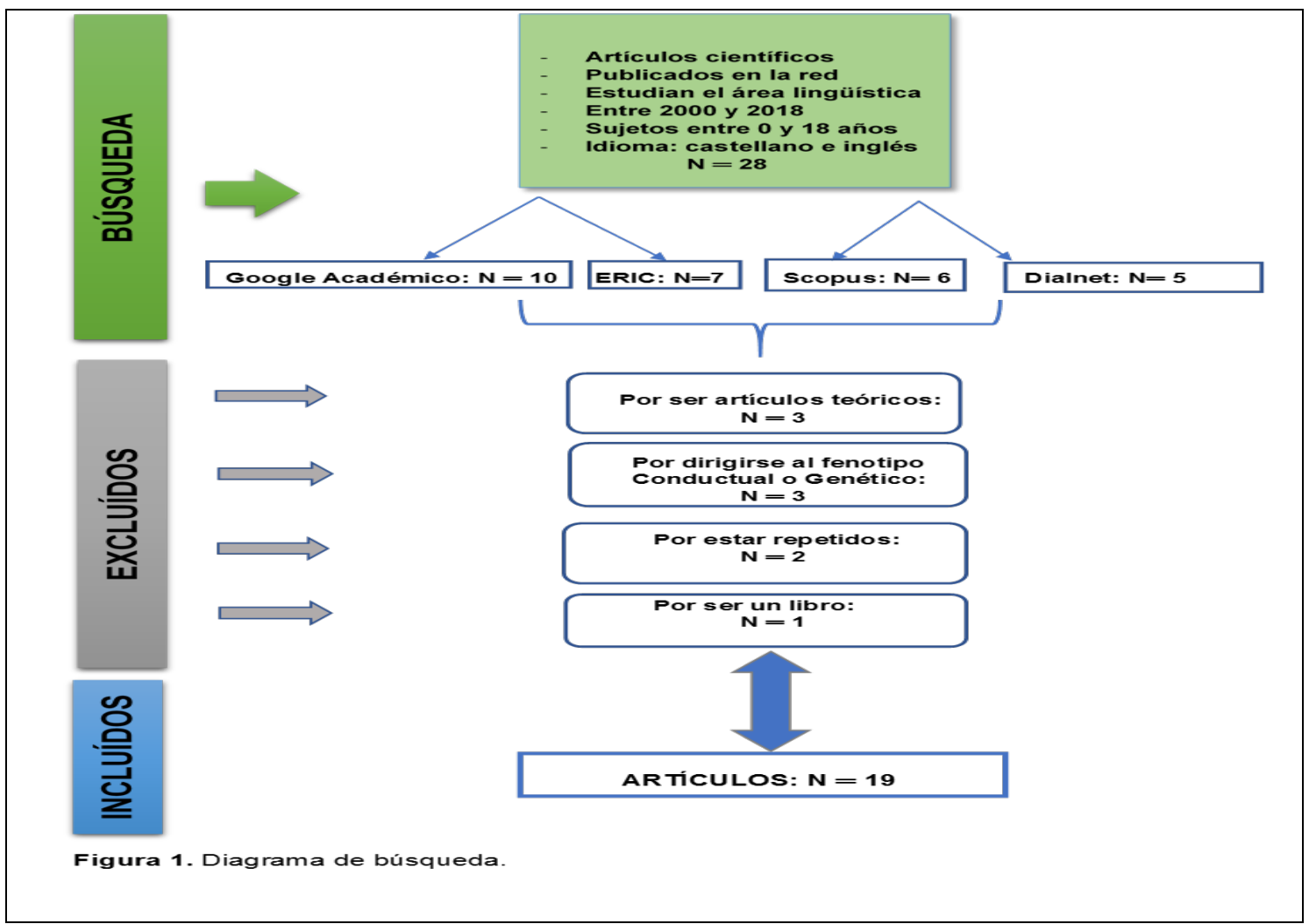




\section{Resultados}

En las siguientes tablas ( 1 y 2 ) se describen los diecinueve artículos seleccionados para esta revisión bibliográfica en base a la siguiente información: autor, año y origen del estudio, objetivos, descripción de la muestra, instrumentos de medida, tipo de evaluación o intervención aplicada y resultados.

Casi la totalidad de los estudios se realizaron entre EEUU y España, concretamente 12 en EE. UU (Roberts et al. 2002; Murphy y Abbeduto, 2007; Roberts et al. 2007; Barnes et al. 2009; Warren et al. 2011; Koover et al. 2012; Sterling y Abbeduto, 2012; Haebig et al. 2016; Klusek et al. 2016; McDuffie et al. 2016; Friedman et al. 2018; Nelson et al. 2018) y 5 en España (Robles, 2011; Robles et al. 2013; Herráiz et al. 2014; Puente et al. 2017; Huelmo et al. 2016).

Respecto al tipo de estudio, se observa que son dos las tendencias principales, por un lado, los estudios comparativos entre diferentes trastornos o en ausencia de éstos y por otro, los estudios de campo, repartidos casi equitativamente a la mitad. En concreto, los estudios de campo encontrados son 10 (Roberts et al. 2002; Murphy y Abbeduto, 2007; Warren et al. 2011; Robles, 2011; Sterling y Abbeduto, 2012; Robles et al. 2013; Badran et al. 2013; Klusek et al. 2016; McDuffie et al. 2016; Nelson et al. 2018) frente a los 9 estudios comparativos (Comblain y Elbouz, 2002; Roberts et al. 2007; Barnes et al. 2009; Koover et al. 2012; Herráiz et al. 2014; Haebig et al. 2016; Puente et al. 2017; Huelmo et al. 2016; Friedman et al. 2018).

Con relación a la edad de la muestra prevalecen dos rangos que son, por una parte, aquella que acoge la etapa de Educación Infantil y por otra, la que acoge Educación Primaria y Secundaria. Concretamente en 4 de los estudios la muestra recoge participantes entre los 0 y los 6 años (Roberts et al. 2002; Warren et al. 2011; Robles, 2011; Robles et al. 2013) y en 11 de los estudios entre los 6 y los 18 años (Comblain y Elbouz, 2002; Murphy y Abbeduto, 2007; Roberts et al. 2007; Sterling y Abbeduto, 2012; Herráiz et al. 2014; Klusek et al. 2016; McDuffie et al. 2016; Puente et al. 2017; Huelmo et al. 2016; Friedman et al. 2018; Nelson et al. 2018). El resto de los estudios, aunque están dentro de este grupo de edad, no se adecuan a ninguno de los dos rangos.

Además, respecto al género de la muestra, es destacable que únicamente en 1 artículo se estudia el femenino de manera exclusiva (Sterling y Abbeduto, 2012) frente a los 11 que estudian el género masculino (Comblain y Elbouz, 2002; Roberts et al. 2002; Roberts et al. 2007; Barnes et al. 2009; Robles, 2011; Koover et al. 2012; Robles et al. 2013; Badran et al. 2013; Herráiz et al. 2014; Haebig et al. 2016; Friedman et al. 2018). Por tanto, el resto de las investigaciones estudian tanto el género femenino como el masculino, aunque no distribuido equitativamente.

En cuanto a la metodología de los estudios es remarcable que en 3 ésta es únicamente familiar (Warren et al. 2011; Klussek et al. 2016; Nelson et al. 2018) mientras que en otros 6 se añade la metodología escolar (Robles, 2011; Sterling y Abbeduto 2012; Robles et al. 2013; Badran et al. 2013; Herráiz et al. 2014; McDuffie et al. 2016). El resto de las investigaciones se desarrollan únicamente en el ámbito escolar.

Por otro lado, de los diecinueve artículos encontrados 15 son de evaluación (tabla 1) y 4 son de intervención (tabla 2).Borra y escribe los resultados de forma concisa y clara. Se redactarán de modo que no haya duplicación ni repetición innecesaria de información en el texto, figuras o tablas. 
Tabla 1. Artículos relacionados con el perfil de los niños con síndrome $x$ frágil y el lenguaje

\begin{tabular}{|c|c|c|c|c|c|}
\hline Autores, año y origen & Objetivos & Muestra & Instrumentos & Evaluación & Resultados \\
\hline $\begin{array}{l}\text { Comblain y Elbouz. } \\
\text { (2002, Bélgica) }\end{array}$ & $\begin{array}{l}\text { Evaluar la capacidad del SXF como } \\
\text { orador y oyente en tareas de } \\
\text { comunicación referencial, mediante } \\
\text { la interacción con un adulto o un } \\
\text { niño de DT o DI. }\end{array}$ & $\begin{array}{l}4 \text { SXF } \\
\text { DI } \\
\text { DT niños } \\
\text { DT adultos } \\
S=0 \\
E=6-12 \text { años. }\end{array}$ & - PPVT & $\begin{array}{l}\text { Diseño experimental clásico, mensaje espontáneo: } \\
\text { el oyente colocaba bloques en la posición descrita } \\
\text { por el orador, diferenciando por color, forma y } \\
\text { tamaño o combinación de dos variables. }\end{array}$ & $\begin{array}{l}\text { Como oradores dan mensajes insuficientes en tareas de } \\
\text { combinación. Como oyentes, si el adulto da un mensaje } \\
\text { completo, más del } 80 \% \text { de respuestas son correctas y con un } \\
\text { niño de Dl el } 50 \% \text {. Con mensaje incompleto o niño DT el } \\
\text { rendimiento disminuye. }\end{array}$ \\
\hline $\begin{array}{l}\text { Roberts, Mirret, Anderson, } \\
\text { Burchinal y Neebe. } \\
\text { (2002, EEUU) }\end{array}$ & $\begin{array}{l}\text { Examinar el perfil comunicativo y } \\
\text { conductual en niños SXF que están } \\
\text { en una etapa de desarrollo } \\
\text { temprano. }\end{array}$ & $\begin{array}{l}\mathrm{G}=22 \mathrm{SXF} \\
\mathrm{S}=\hat{O} \\
\mathrm{E}=21-77 \mathrm{~m} . \\
\text { (desarrollo<28 m). }\end{array}$ & $\begin{array}{l}\text { - Battelle. } \\
\text { - CSBS } \\
\text {-Escalas del Desarrollo } \\
\text { del Lenguaje. }\end{array}$ & $\begin{array}{l}\text { Se evaluaron los modos y funciones de } \\
\text { comunicación, reciprocidad, señalización social } \\
\text { afectiva y las conductas simbólicas de juego. }\end{array}$ & $\begin{array}{l}\text { La variabilidad individual dificultó establecer un perfil } \\
\text { comunicativo, mostraron fortalezas en comunicación y } \\
\text { debilidades en el resto. A mejor comunicación y reciprocidad } \\
\text { mejor comprensión verbal y desarrollo del lenguaje. }\end{array}$ \\
\hline $\begin{array}{l}\text { Murphy y Abbeduto. } \\
\text { (2007, EEUU) }\end{array}$ & $\begin{array}{l}\text { Analizar la relación en el SXF entre } \\
\text { el género, la perseverancia verbal; } \\
\text { habilidades cognitivas y lingüísticas } \\
\text { y lenguaje contextual. }\end{array}$ & 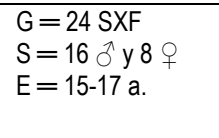 & $\begin{array}{l}\text { - Stanford-Binet Intelligence } \\
\text { - OWLS } \\
\text { - Frog Goes to Dinner } \\
\text { - Software SALT }\end{array}$ & $\begin{array}{l}\text { Se evaluó la auto-repetición de palabras, frases, } \\
\text { locuciones y temas. El lenguaje evaluado fue } \\
\text { espontáneo en } 2 \text { contextos lingüisticos: narración } \\
\text { del libro y conversación guiada. }\end{array}$ & $\begin{array}{l}\text { Ellas tenían un } \mathrm{Cl} \text { y habilidades de lenguaje más altas. Ellos } \\
\text { repitieron más en conversación. Todos repitieron según el } \\
\text { contexto de la narración. Ellos se desenvuelven peor en } \\
\text { pragmática y respuesta fisiológica en interacción social. }\end{array}$ \\
\hline $\begin{array}{l}\text { Roberts, et al. } \\
\text { (2007, EEUU) }\end{array}$ & $\begin{array}{l}\text { Establecer las habilidades del } \\
\text { discurso conversacional. }\end{array}$ & $\begin{array}{l}N=105 \hat{} \\
28 \text { SXF } \\
26 \text { SXF+TEA } \\
29 \text { SD } \\
22 \text { DT } \\
E=\geq 16\end{array}$ & $\begin{array}{l}\text {-Leiter-R } \\
\text {-ADOS }\end{array}$ & $\begin{array}{l}\text { Se evaluó la interacción conversacional, para } \\
\text { extraer la capacidad de mantener y cambiar de } \\
\text { tema en actividades estructuradas (juego libre, } \\
\text { simulación cumpleaños y lectura de cuentos). }\end{array}$ & $\begin{array}{l}\text { Los SXF+TEA produjeron más discursos no contingentes; } \\
\text { todos los grupos mantuvieron el tema de conversación entre } \\
\text { el } 84-88 \% \text { de los turnos, siendo la variable de mantenimiento } \\
\text { adecuado de conversación la mejor puntuada. }\end{array}$ \\
\hline $\begin{array}{l}\text { Barnes, et al. } \\
\text { (2009, EEUU) }\end{array}$ & $\begin{array}{l}\text { Determinar la precisión fonológica y } \\
\text { la inteligibilidad del habla. }\end{array}$ & $\begin{array}{l}N=142 \hat{\sigma} \\
32 \text { SXF } \\
31 \text { SXF+TEA } \\
34 \text { SD } \\
45 \text { DT } \\
E=3-16\end{array}$ & $\begin{array}{l}\text {-Leiter-R } \\
\text {-ADOS } \\
\text {-PEPPER }\end{array}$ & $\begin{array}{l}\text { Se evaluó la emisión y aplicación de palabras y } \\
\text { frases en el habla para calcular la exactitud } \\
\text { fonológica y la inteligibilidad, según un mínimo de } \\
100 \text { palabras inteligibles, excluyendo deletreos, } \\
\text { interjecciones, y palabras sueltas. }\end{array}$ & $\begin{array}{l}\text { Los SXF (con o sin TEA) no difirieron de los de DT en la } \\
\text { precisión fonológica, pero produjeron menos palabras } \\
\text { inteligibles, equiparándose a los SD que tuvieron } \\
\text { calificaciones más bajas que el resto de los grupos. }\end{array}$ \\
\hline $\begin{array}{l}\text { Warren, Brady, Sterling, } \\
\text { Fleming y Marquis. } \\
\text { (2011, EEUU) }\end{array}$ & $\begin{array}{l}\text { Hallar la relación entre la capacidad } \\
\text { de respuesta materna temprana y } \\
\text { los resultados posteriores de la } \\
\text { comunicación infantil en los hijos } \\
\text { con SXF. }\end{array}$ & $\begin{array}{l}\mathrm{N}=55 \text { parejas } \\
\text { madre e hijo/a. } \\
\mathrm{S}=44 \text { ○ } \mathrm{y} 11 \text { } \\
\mathrm{E}=11-76 \mathrm{~m} . \\
\mathrm{G}=\mathrm{SXF} \sin \mathrm{TEA}\end{array}$ & $\begin{array}{l}\text {-CARS } \\
\text {-Escala de Aprendizaje } \\
\text { Temprano. } \\
\text {-Escala de Inteligen. Adulta } \\
\text {-Software Noldus. }\end{array}$ & $\begin{array}{l}\text { Se evaluó y grabó el lenguaje en } 4 \text { contextos } \\
\text { (leyendo un libro juntos, preparando y comiendo un } \\
\text { bocadillo, juegos no estructurados y habla de } 30^{\prime} \\
\text { en contexto natural). Se codificó la conducta por } \\
\text { capacitación individual. }\end{array}$ & $\begin{array}{l}\text { La capacidad de respuesta materna temprana predijo el nivel } \\
\text { del lenguaje infantil, principalmente cuando las madres se } \\
\text { involucraron en niveles más altos de comunicación y los } \\
\text { niños eran de edad inferior a los } 36 \text { meses. }\end{array}$ \\
\hline $\begin{array}{l}\text { Koover, McDuffie, Abbeduto } \\
\text { y Brown. } \\
\text { (2012, EEUU) }\end{array}$ & $\begin{array}{l}\text { Comparar el lenguaje expresivo en } \\
\text { tres grupos. }\end{array}$ & $\begin{array}{l}N=57 \\
S=\widehat{O} \\
27 \text { SXF (E=10-17) } \\
15 \text { SD (E= 10-17) } \\
15 D T(E=3-6) \\
\text { igualados a grupos } \\
\text { clínicos en edad } \\
\text { lingüística. }\end{array}$ & $\begin{array}{l}\text {-Leiter-R } \\
\text {-SALT } \\
\text {-Frog Goes to Dinner. }\end{array}$ & $\begin{array}{l}\text { Se evaluó el lenguaje, la diversidad léxica y fluidez; } \\
\text { cantidad de comunicación (MLCU) en una } \\
\text { entrevista de } 10 \text { min sobre sus intereses y la } \\
\text { narración del libro. }\end{array}$ & $\begin{array}{l}\text { Los SXF tuvieron menor MLCU y diversidad léxica que los de } \\
\text { DT. } \\
\text { Los SD produjeron un MLCU más bajo. Diferencias entre los } \\
\text { grupos clínicos y el de DT en minutos usados, MLCU y la } \\
\text { fluidez. Los SXF mostraron relación entre la severidad del } \\
\text { autismo y el número de expresiones producidas en la } \\
\text { conversación. }\end{array}$ \\
\hline $\begin{array}{l}\text { Sterling y Abbeduto. } \\
\text { (2012, EEUU) }\end{array}$ & $\begin{array}{l}\text { Evaluar el lenguaje receptivo y } \\
\text { expresivo en mujeres SXF en } \\
\text { vocabulario y sintaxis. }\end{array}$ & $\begin{array}{l}\mathrm{G}=21 \mathrm{SXF} \\
\mathrm{S}=+ \\
\mathrm{E}=7-15 \\
\mathrm{E} \text { media verbal } \\
11,8\end{array}$ & $\begin{array}{l}- \text {-PPVT-III } \\
\text {-PPVT-IV } \\
\text {-Leiter-R }\end{array}$ & $\begin{array}{l}\text { Se evaluó la sintaxis con entrevista de preguntas } \\
\text { abiertas, verificada por un segundo examinador. La } \\
\text { mayoría en el laboratorio (el resto en sus casas). }\end{array}$ & $\begin{array}{l}\text { Un } 14 \% \text { casi no tenían vocabulario y } 14 \% \text { en el límite } \\
\text { lingüístico. Un } 45 \% \text { tenía DI y un } 40 \% \text { estaban en el límite. } \\
\text { Conversaban con lenguaje complejo, pero en } 2 \text { de ellas fue } \\
\text { inferior a su edad. EI Cl medio indicaba DI moderada (70). }\end{array}$ \\
\hline
\end{tabular}




\begin{tabular}{|c|c|c|c|c|c|}
\hline Autores, año y origen & Objetivos & Muestra & Instrumentos & Evaluación & Resultados \\
\hline $\begin{array}{l}\text { Badran, Abulnasr } \\
\text { y Nasser. } \\
\text { (2013, Egipto) }\end{array}$ & $\begin{array}{l}\text { Estudiar el efecto de las infecciones } \\
\text { recurrentes del oído medio (otitis) } \\
\text { en el perfil lingüístico de niños SXF } \\
\text { sin discapacidad auditiva. }\end{array}$ & $\begin{array}{l}\mathrm{N}=30 ; 2 \text { grupos } \\
\mathrm{A} .15 \mathrm{SXF} \text { con } \\
\text { antecedentes. } \\
\text { B. } 15 \mathrm{SXF} \text { sin } \\
\text { antecedentes. } \\
\mathrm{S}=0 \\
\mathrm{E}=4-10\end{array}$ & $\begin{array}{l}\text { - DSM-IV } \\
\text { - Stanford-Binet. } \\
\text { - Test de lengua árabe }\end{array}$ & $\begin{array}{l}\text { Se evaluó mediante entrevista paterna, examen } \\
\text { neurológico, audiológico, tracto vocal y análisis } \\
\text { genético. Se evaluó la inteligibilidad } \\
\text { y habla espontánea según sus oraciones. }\end{array}$ & $\begin{array}{l}\text { El grupo A mostró mayores problemas en todas las } \\
\text { habilidades del lenguaje. }\end{array}$ \\
\hline $\begin{array}{l}\text { Herráiz, Fernández, Pastor, } \\
\text { Puchol y Sanz. } \\
\text { (2014, España) }\end{array}$ & $\begin{array}{l}\text { Comparar el SXF con trastornos } \\
\text { comórbidos en funcionamiento } \\
\text { ejecutivo y lenguaje. }\end{array}$ & $\begin{array}{l}\mathrm{N}=50^{\wedge} \\
1 \text { SXF } \\
\text { 1DI } \\
\text { 1TEA } \\
\text { 1TDAH } \\
\text { 1 DT } \\
\mathrm{E}=8-9\end{array}$ & $\begin{array}{l}\text { - PPVT-III } \\
\text { - Velocidad de Denominación } \\
\text { - Comprensión Instrucciones } \\
\text { (NEPSY II). } \\
\text { - Expresión } \\
\text { - Adivinanzas (WPPSI III). }\end{array}$ & $\begin{array}{l}\text { Se evaluó individualmente. } \\
\text { Se comparó las puntuaciones directas de cada } \\
\text { sujeto en base al sujeto control de DT. }\end{array}$ & $\begin{array}{l}\text { El SXF se comportó igual que el DI y el TEA en comprensión } \\
\text { de instrucciones y razonamiento verbal. En fluidez inducida } \\
\text { se comportó igual que el DI y mostró menos destreza en } \\
\text { fluidez verbal libre y léxico que los demás trastornos. }\end{array}$ \\
\hline $\begin{array}{l}\text { Haebig, Sterling y Hoover. } \\
\text { (2016, EEUU) }\end{array}$ & $\begin{array}{l}\text { Determinar los procesos del } \\
\text { lenguaje subyacente afectados por } \\
\text { los trastornos del desarrollo. }\end{array}$ & $\begin{array}{l}\mathrm{N}=56 \\
17 \mathrm{SXF}(\mathrm{E} \mathrm{11,9)} \\
20 \mathrm{TEL}(\mathrm{E} \mathrm{4,9)} \\
19 \mathrm{DT}(\mathrm{E} \mathrm{3,3)} \\
\mathrm{S}=\mathrm{O}^{-}\end{array}$ & $\begin{array}{l}\text { - PPVT } \\
\text { - Prueba de Deterioro } \\
\text { Gramatical Temprano. } \\
\text { - Leiter }\end{array}$ & $\begin{array}{l}\text { Se evaluó el lenguaje mediante comparación } \\
\text { cuantitativa y cualitativa según la interacción } \\
\text { niñolexaminador. A través de una conversación (10 } \\
\text { min.) con temas y preguntas abiertas. }\end{array}$ & $\begin{array}{l}\text { Los SXF mostraron buena interacción entre su gramática y } \\
\text { sintaxis, el desempeño fue inferior a las expectativas y a la } \\
\text { sintaxis receptiva, dando respuestas no calificables que } \\
\text { manifestaron su déficit pragmático. }\end{array}$ \\
\hline $\begin{array}{l}\text { Klusek, McGrath, Abbeduto } \\
\text { y Roberts. } \\
\text { (2016, EEUU) }\end{array}$ & $\begin{array}{l}\text { Conocer la interacción de la } \\
\text { pragmática en madres y el lenguaje } \\
\text { de sus hijos (vocabulario, sintaxis y } \\
\text { lectura). }\end{array}$ & $\begin{array}{l}\mathrm{N}=27 \text { parejas de } \\
\text { madre e hijo } \mathrm{SXF} . \\
\mathrm{S}=+\hat{0} \\
\mathrm{E}=17,9 \text { (media) }\end{array}$ & $\begin{array}{l}\text {-PPVT } \\
\text {-Escala pragmática. } \\
\text {-Escala gramática de lenguaje } \\
\text { oral. } \\
\text {-Reading Mastery Tests-III }\end{array}$ & $\begin{array}{l}\text { Se evaluó la pragmática materna (conversaciones } \\
\text { de } 20 \text { min.) y la capacidad de (interacciones } \\
\text { madre-hijo como solicitudes de cumplimientos). }\end{array}$ & $\begin{array}{l}\text { Las dificultades del lenguaje pragmático materno se } \\
\text { asociaron con el vocabulario receptivo de los niños (con } \\
\text { mayor dificultad) y las habilidades de sintaxis expresiva. }\end{array}$ \\
\hline $\begin{array}{l}\text { Puente, Alvarado, Jiménez y } \\
\text { Martinez. } \\
\text { (2017, Chile y España) }\end{array}$ & $\begin{array}{l}\text { Comparar la alfabetización, el } \\
\text { conocimiento fonológico y el } \\
\text { rendimiento lector del grupo SXF } \\
\text { con el SD. }\end{array}$ & 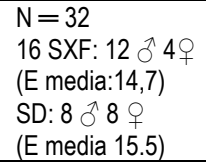 & $\begin{array}{l}\text {-PPVT-III } \\
\text {-MSCA } \\
\text {-Prueba de Dominio Lector. } \\
\text {-PROBALES }\end{array}$ & $\begin{array}{l}\text { Se evaluó su habilidad en conciencia fonológica, } \\
\text { reconocimiento y comprensión. } \\
\text {. }\end{array}$ & $\begin{array}{l}\text { No hubo diferencias en vocabulario y en fonología. } \\
\text { Obtuvieron menor puntuación en reconocimiento y } \\
\text { comprensión. Hubo mejor desempeño en inteligencia verbal y } \\
\text { menor en interpretar oraciones o escuchar, reflejando su } \\
\text { débil pragmática. }\end{array}$ \\
\hline $\begin{array}{l}\text { Huelmo, Martínez y Diez. } \\
\text { (2017, España) }\end{array}$ & $\begin{array}{l}\text { Establecer un perfil fonológico en } \\
\text { base a los errores cometidos. }\end{array}$ & $\begin{array}{l}\mathrm{N}=46 \\
6 \mathrm{SXF}, \text { Jadultos } \\
\text { (E cronológica } 44, \\
\text { verbal } 8,9) \\
40 \mathrm{DT}, 20 \text { ○े } 20 \text { \% } \\
(\mathrm{E}=5,8)\end{array}$ & $\begin{array}{l}\text { - PPVT } \\
\text { - CHAT } \\
\text { - Software CLAN }\end{array}$ & $\begin{array}{l}\text { Se evaluó mediante el cálculo del índice de error } \\
\text { fonológico (IE) sobre el total de palabras según: } \\
\text { estructura de sílaba (ES), sustitución (ST), omisión } \\
\text { (OM) y asimilación (SM). }\end{array}$ & $\begin{array}{l}\text { EI SXF erró más en (ES) y (SM) por su dificultad en la } \\
\text { coarticulación de grupos consonánticos. La producción y } \\
\text { diversidad léxica es menor en el SXF y el IE mayor, pero sin } \\
\text { mucha diferencia. Los errores fonológicos en la diferencia } \\
\text { entre grupos no fueron suficiente para explicar el habla. }\end{array}$ \\
\hline $\begin{array}{l}\text { Friedman, Sterling y Barton. } \\
\text { (2018, EEUU) }\end{array}$ & $\begin{array}{l}\text { Evaluar la relación entre la elusión } \\
\text { de la mirada y el lenguaje } \\
\text { perseverante. }\end{array}$ & $\begin{array}{l}\mathrm{N}=20 \\
\mathrm{SXF}+\mathrm{TEA}(\mathrm{E} 12) \\
\mathrm{TEA}(\mathrm{E} 13) \\
\mathrm{S}=\hat{0}\end{array}$ & $\begin{array}{l}\text {-Leiter } \\
\text {-ADOS } \\
\text {-SALT } \\
\text {-Software de video } \\
\text {-Proceder }\end{array}$ & $\begin{array}{l}\text { Se evaluó lenguaje y evitación de la mirada } \\
\text { mediante conversación semiestructurada (10 min), } \\
\text { preguntas abiertas sobre intereses personales (sin } \\
\text { pedir contacto visual). }\end{array}$ & $\begin{array}{l}\text { Los SXF+TEA dirigieron la mirada menos tiempo }(17.92 \% \\
\text { frente al } 22.18 \%) \text { y perseveraron más. No se encontró } \\
\text { relación entre evitación y perseveración, indicando que una } \\
\text { no aumenta la otra. }\end{array}$ \\
\hline
\end{tabular}


Tabla 2. Artículos relacionados con la intervención de los niños con síndrome x frágil en el lenguaje

\begin{tabular}{|c|c|c|c|c|c|}
\hline Autores, año y origen & Objetivos & Muestra & Instrumentos & Evaluación & Resultados \\
\hline $\begin{array}{l}\text { Robles } \\
\text { (2011, España) }\end{array}$ & $\begin{array}{l}\text { Intervenir individualmente en } \\
\text { lenguaje, motora, perceptiva y } \\
\text { autonomía a un sujeto con } \\
\text { SXF. }\end{array}$ & $\begin{array}{l}\mathrm{N}=1 \mathrm{SXF} \text { con } \\
\text { antecedentes familiares. } \\
\mathrm{S}=\hat{\lambda} \\
\mathrm{E}=10 \mathrm{~m} .\end{array}$ & $\begin{array}{l}\text { Programa de Estimulación del } \\
\text { desarrollo Infantil. }\end{array}$ & $\begin{array}{l}\text { Se intervino con diseño AB, en sesión individual en } \\
\text { casa diariamente. } 1 \text { sesión grupal con padres cada } 2 \\
\text { meses }\end{array}$ & $\begin{array}{l}\text { Emite sonidos vocálicos, dice } 1 \text { palabra bisílaba y } \\
\text { parece reaccionar a palabras familiares y a su nombre. } \\
\text { Mejoró su lenguaje y demás áreas. }\end{array}$ \\
\hline $\begin{array}{l}\text { Robles, Sánchez } \\
\text { y Valencia. } \\
\text { (2013, España) }\end{array}$ & $\begin{array}{l}\text { Intervenir en } 2 \text { casos de SXF } \\
\text { en lenguaje, área motora, } \\
\text { perceptiva y autonomía. Uno } \\
\text { de los casos no se interviene } \\
\text { en el lenguaje. }\end{array}$ & $\begin{array}{l}\mathrm{N}=2 \mathrm{SXF} \text { con } \\
\text { antecedentes familiares. } \\
\mathrm{S}=\widehat{\lambda} \\
\mathrm{E}=15 \mathrm{~m} . \text { ( (sujeto } \mathrm{A}) \\
\quad 26 \mathrm{~m} \text { (sujeto } \mathrm{B})\end{array}$ & $\begin{array}{l}\text { - Escala de Desarrollo Psicomotor de } \\
\text { la Primera Infancia Brunet-Lézine-R. } \\
\text {-Programa de Estimulación del } \\
\text { desarrollo Infantil. }\end{array}$ & $\begin{array}{l}\text { Se intervino mediante diseño de caso único } A B \text {. } \\
3 \text { sesiones semanales para sujeto } B \text { y } 1 \text { para el } A \text {. }\end{array}$ & $\begin{array}{l}\text { Posttest: (A) Emite sonidos vocálicos, } 1 \text { palabra bisílaba, } \\
\text { reacciona a su nombre y gestualiza. (B) Asoció } \\
\text { estímulos auditivos, imitó sonidos y siguió órdenes } \\
\text { sencillas. Consolidó articulación vocálica, aparece } / \mathrm{m}, \mathrm{n} \text {, } \\
\text { t, kl. Más vocabulario Apareció la holofrase para pedir. } \\
\text { Reacciona al lenguaje, pero sin mirar. }\end{array}$ \\
\hline $\begin{array}{l}\text { McDuffie, et al. } \\
\text { (2016, EE. UU) }\end{array}$ & $\begin{array}{l}\text { Analizar si el lenguaje } \\
\text { narrativo implementado por } \\
\text { madres determina el lenguaje } \\
\text { inferencial durante la } \\
\text { intervención compartida. }\end{array}$ & $\begin{array}{l}\mathrm{N}=3 \text { parejas de } \\
\quad \text { madre e hijo } \\
\mathrm{S}=\stackrel{+}{\lambda} \\
\mathrm{E}=10-16 \text { a } \\
\mathrm{G}=\mathrm{SXF}\end{array}$ & $\begin{array}{l}4 \text { libros: examinador Frog Goes to } \\
\text { Dinner y Frog on the Own. Con la } \\
\text { madre: Frog, Where Are You? } \\
\text { One Frog Too Many. }\end{array}$ & $\begin{array}{l}\text { Se intervino individualmente el habla en la narración } \\
\text { compartida. Examinador: el niño miraba el libro y } \\
\text { contaba la historia. Con la madre, lo miraban juntos y } \\
\text { le alentaba a contarla. } \\
12 \text { sesiones. }\end{array}$ & $\begin{array}{l}\text { El habla aumentó. Los hijos podrían considerarse } \\
\text { verbalmente fluidos al producir frases para comunicar } \\
\text { sus deseos y necesidades, pero insuficiente para } \\
\text { participar en conversaciones extensas, contar } \\
\text { experiencia personal o repetir una historia. }\end{array}$ \\
\hline $\begin{array}{l}\text { Nelson, et al. } \\
\text { (2018, EE. UU) }\end{array}$ & $\begin{array}{l}\text { Evaluar si una intervención } \\
\text { implementada por madres } \\
\text { determina el lenguaje } \\
\text { inferencial y espontáneo de } \\
\text { sus hijos SXF durante la } \\
\text { narración compartida. }\end{array}$ & $\begin{array}{l}\mathrm{N}=19 \text { parejas } \\
\text { madres e hijos } \\
\mathrm{E}=10-17 \text {. } \\
\mathrm{G}=\mathrm{SXF} \\
\rightarrow \text { Excepto } 3, \text { los hijos } \\
\text { recibían servicios de } \\
\text { lenguaje específico } \\
\text { durante el estudio. }\end{array}$ & $\begin{array}{l}\text { Digitalización de } 2 \text { libros ilustrados } \\
\text { infantiles similares y guiones para } \\
\text { madres. } \\
\text { Equipo de videoconferencia con } \\
\text { portátil y auricular. }\end{array}$ & $\begin{array}{l}\text { En la pre-intervención, las madres tuvieron } 1 \text { sesión } \\
\text { de apoyo conductual y } 2 \text { de estrategias lingüísticas. } \\
\text { En la intervención resumían el libro dando } \\
\text { vocabulario, gramática y preguntas. } \\
24 \text { sesiones. }\end{array}$ & $\begin{array}{l}\text { Las madres aumentaron su uso de estrategias, } \\
\text { aumentaron la frecuencia y la duración de las } \\
\text { conversaciones relacionadas con la historia. Se } \\
\text { observaron efectos generalizados de la intervención } \\
\text { sobre la diversidad léxica y la complejidad gramatical. }\end{array}$ \\
\hline
\end{tabular}




\section{Discusión}

Con relación a las dimensiones del lenguaje analizadas en las investigaciones revisadas, un primer conjunto de estudios coincide en afirmar que el área pragmática es la más perjudicada en los niños y niñas con SXF. Concretamente los resultados revelan que en calidad de oradores su rendimiento es inferior ya que sus mensajes espontáneos son insuficientes en tareas de comunicación referencial o en interacciones conversacionales a causa de entre otros factores su ansiedad ante determinadas situaciones sociales o su frecuente evitación de la mirada. Además, esta área es incluso inferior en el género masculino y se refleja en los hijos de madres con SXF cuyas dificultades pragmáticas les afectan no sólo en este componente sino también a nivel de léxico y de sintaxis (Comblain y Elbouz, 2002; Murphy y Abbeduto, 2007; Koover et al. 2012; Klusek et al. 2016; McDuffie et al. 2016; Friedman et al. 2018). Sin embargo 1 de las investigaciones considera que su mantenimiento del tema en la conversación es bueno en base a diversas variables de calidad de tema estudiadas (Roberts et al. 2007).

En cuanto a la adquisición y el desarrollo del léxico un grupo de estudios coincide en afirmar que tanto la diversidad léxica como el número de palabras inteligibles en el vocabulario de los niños SXF es menor comparada con otros niños, resultando insuficiente para participar en conversaciones con cierto grado de complejidad como contar una experiencia personal o repetir una historia coherente que se ha escuchado (Barnes et al. 2009; Koover et al. 2012; Herráiz et al. 2014; McDuffie et al. 2016). Sin embargo, también afirman que se les puede considerar verbalmente fluidos para comunicar sus necesidades o deseos diarios y que por tanto la diferencia no es suficientemente significativa para comprometer la inteligibilidad de su habla (McDuffie et al. 2016; Huelmo et al. 2017).

Respecto al área fonológica, determinados estudios revelan que la precisión fonológica del SXF está bastante preservada en comparación con otras habilidades lingüísticas como son la pragmática, siendo suficientemente competente pues los errores fonológicos que cometen no son lo bastante numerosos para entorpecer su habla (Puente et al. 2017; Huelmo et al. 2017).

Con relación a la sintaxis, los estudios revelan por un lado que está más atrasada en comparación con otros niños, principalmente en lo que a recepción se refiere y por otro, que ésta se ve afectada por las dificultades pragmáticas maternas (Haebig et al. 2016; Klusek et al. 2016). Una de las investigaciones afirma que dicha dificultad sintáctica, en el caso de las niñas, no les perjudica a la hora de mantener un lenguaje complejo (Sterling y Abbeduto, 2012).

Asimismo, ciertos autores confirman la existencia de factores secundarios que pueden incrementar la dificultad lingüística en todos sus componentes, como son las infecciones recurrentes del Oído Medio en aquellos que sin tener una discapacidad auditiva diagnosticada son más propensos a éstas, revelando una mayor probabilidad del SXF debido a la morfología atípica de su oído externo (Badran et al. 2013).

Pese a todo lo anterior, cabe remarcar que debido a la variabilidad individual de los niños y niñas con SXF resulta difícil establecer un perfil comunicativo definitivo (Barnes et al. 2002); siendo recomendable la evaluación e intervención a partir de edades tempranas.

En el caso de las evaluaciones, se extrae resultados superiores, principalmente cuando el niño tiene menos de 3 años, de acuerdo con (Warren et al. 2011). En cuanto 
a las intervenciones, por un lado, se confirma la efectividad de los Centros Especializados en la Atención Temprana, concretamente de los CADIT, ya que según (Robles, 2011; Robles et al. 2013) los casos intervenidos mejoraron en todas las áreas evaluadas, siendo estas el lenguaje, la motora, la perceptiva y la autonomía. Por otro lado, se confirma también la eficacia de la narración compartida entre madres e hijos, pues de acuerdo con (McDuffie et al. 2016) a través de ésta mejoró el habla en ambos. De la misma manera, las madres aumentaron en su uso de estrategias, la frecuencia y la duración de las conversaciones relacionadas con la historia según (Nelson et al. 2018), con el soporte de un entrenamiento previo en el uso de preguntas abiertas y entonación.

Con relación a la tarea de los logopedas y maestros de audición y lenguaje (AyL), éstos deben hacer uso de los instrumentos y ejercicios que permitan al alumnado SXF mejorar y optimizar sus habilidades comunicativas, enfatizando en la mejora de la articulación y pronunciación, en pro de una mayor comprensión, aumentando su vocabulario y la extensión de sus frases o conversaciones y propiciando el lenguaje espontáneo. Sin olvidar que, la terapia del lenguaje debe tener en cuenta el alto nivel de ansiedad que denotan los niños al adaptarse a cambios en el ambiente o transiciones y al interactuar o acceder a nueva información, tal y como apunta Fürgang (2001).

En definitiva, el trabajo aquí presentado plantea las dificultades que manifiestan los niños y niñas con SXF en el ámbito lingüístico y todas las dimensiones que éste acoge, para tenerlas en cuenta en un futuro a la hora de elaborar propuestas de intervención con este alumnado. Confirma, por una parte, la importancia de realizar evaluaciones para saber la línea base de la que parten en los diversos parámetros del lenguaje y la comunicación, y por otra, la efectividad de intervenir a edades tempranas con el objetivo de desarrollar lo más pronto posible un uso adecuado del lenguaje con las mínimas dificultades y adecuarlo a los contextos discursivos que requiere la comunicación. Asimismo, muestra la eficacia de determinados métodos o técnicas de intervención, como son la conversación guiada a través de preguntas y listas con temas de su interés o la narración de libros ilustrados que al requerir de una mayor reciprocidad entre examinador y niño les resulta más desafiante por el hecho de tener, como mencionan muchos de los autores, una pragmática más debilitada y sin embargo los resultados extraídos son positivos. Se observa también, la importancia de realizar tanto evaluaciones a las madres con SXF como intervenciones compartidas entre madre e hijo, ya que en las primeras se confirma que las dificultades lingüísticas en las progenitoras se trasladan al hijo e influyen sobre su desarrollo comunicativo y las segundas muestran una mejora de los resultados en base al entrenamiento previo con las madres en la narración implementada. Por tanto, todas las observaciones y resultados aquí expuestos pueden ser útiles a la hora de identificar aspectos de las habilidades lingüísticas y comunicativas que puedan utilizarse para futuras intervenciones con garantías de éxito.

A partir de lo anterior y de cara a futuras intervenciones, sería recomendable profundizar más en ciertos componentes lingüísticos como son la pragmática que es donde más dificultad encuentran los niños y niñas con SXF. Se buscaría también diversificar los instrumentos de evaluación y alargar el período de intervención especialmente en aquella basada en la narración compartida. Además, se debería tener en cuenta la muestra para ampliarla a un mayor estudio del trastorno en las niñas, ya que la ausencia de su investigación de manera exclusiva dificulta establecer su perfil lingüístico. 


\section{Referencias}

Los artículos incluidos en la revisión están marcados con un asterisco $\left({ }^{\star}\right)$.

Artigas, J., Gabau, E. y Guitart, M. (2006). Fenotipos conductuales en el retraso mental de origen genético. Revista de Neurología, 42(1) ,15-19.

*Badran, HS., Abulnasr, KM., \& Nasser, SAEH. (2013). Effect of recurrent otitis media on language profile in children with fragile $x$ syndrome. Clinical Medicine Insights: Ear, Nose and Throat, 6, CMENT-S11157.

*Barnes, E., Roberts J., Long, S., Martin, G., Berni, M., Mandulak, K., \& Sideris, J. (2009). Phonological Accuracy and Intelligibility in Connected Speech of Boys with Fragile $X$ Syndrome or Down. Journal of Speech Language and Hearing Resource, 52(4), 1048-1061.

Brun, C. y Artigas, J. (2001). Aspectos psicolingüísticos en el Síndrome del cromosoma $X$ frágil. Revista de neurología, 33(1), 29- 32.

*Comblain, A., \& Elbouz, M. (2002). The Fragile-X Syndrome: What about the Deficit in the Pragmatic Component of Language? Journal of Cognitive Education and Psychology, 2(3), 29-58.

Ferrando, MT., Banús, P. y López, G. (2003). Aspectos cognitivos $y$ del lenguaje en niños con síndrome $X$ frágil. Revista de Neurología, 36(1), 137-142.

*Friedman, L., Sterling, A., \& Barton, A. (2018). Gaze avoidance and perseverative language in fragile $X$ syndrome and autism spectrum disorder: brief report. Developmental neurorehabilitattion, 21(2), 137-140.

Fu, YH., Kuhl, D., Pizzuti, A., Pieretti, M., Sutcliffe, J., Richards, S., ..., \& Caskey, C. (1991). Variation of the CGG repeat at the fragile $X$ site results in genetic instability: resolution of the Sherman paradox. Cell, 67, 1047-1058.

Fürgang, R. (2001). La terapia del lenguaje en el síndrome X frágil. Revista de neurología, 33(1), 82-87.

García, G. Rigau, E. y Artigas, J. (2006). Autismo en el Síndrome X Frágil. Revista de Neurología, 42(2), 95-98.

*Haebig, E., Sterling, A., \& Hoover, J. (2016). Examining the language phenotype in children with typical development, specific language impairment and Fragile $X$ Syndrome. Journal of Speech, Language, and Hearing Research, 59, 1046-1058.

Hagerman, RJ., Leehey, M., Heinrichs, W., Tassone, F., Wilson, R., Hills, J., ..., \& Hagerman PJ. (2001). Intention tremor, parkinsonism, and generalized brain atrophy in male carriers of fragile X. Neurology, 57, 127-130.

*Herráiz, E., Fernández, M.I., Pastor, G., Puchol, I. y Sanz, P. (2014). Estudio sobre el lenguaje y las funciones ejecutivas de un caso de Síndrome X Frágil. International Journal of Developmental and Educational Psychology, 3(1), 213-222.

*Huelmo, J., Martínez, V. y Diez, E. (2017). Evaluación de perfiles fonológicos en el Síndrome $X$ frágil mediante índices de error. International Journal of Developmental and Educational Psychology, 1(3), 67-76.
*Klusek, J., McGrath, S., Abbeduto, L., \& Roberts, J. (2016). Pragmatic language features of mothers with the FMR1 premutation are associated with the language outcomes of adolescents and young adults with Fragile $X$ Syndrome. Journal of Speech, Language, and Hearing Research, 59, 4961.

*Kover, S., McGrath, S., Abbeduto, L., \& Roberts, J. (2012). Effects of sampling context on spontaneous expressive language in males with Fragile $\mathrm{X}$ Syndrome or Down syndrome. Journal of Speech, Language, and Hearing Research, 55, 1022-1038.

Lubs, HA. (1969). A marker X chromosome. American Journal of Human Genetics 21, 231-244.

Martin, JP., \& Bell J. (1943). A pedigree of mental defect showing sex-linkage. Journal of Neurology and Psychiatry, 6, 154-157.

*McDuffie, A., Machalicek, W., Bullard, L., Nelson, S., Mello, M., Tempero, R., Castignetti, N., \& Abbeduto, L. (2016). A Spoken-Language Intervention for School-Aged Boys with Fragile X Syndrome. American Journal on Intellectual and Developmental Disabilities, 121(3), 236-265.

*Murphy, MM., \& Abbeduto, L. (2007). Gender differences in repetitive language in fragile $X$ sindrome. Journal of intellectual disability research, 51(5), 387-400.

Murray, A., Webb, J., Grimley, S., Conway, G., \& Jacobs, P. (1998). Studies of FRAXA and FRAXE in women with premature ovarian failure. Journal of Medical Genetics, 35 , 637-640.

*Nelson, S., McDuffie, A., Banasik, A., Tempero, R., Thurman, A. $\&$ Abbeduto, L. (2018). Inferential language use by schoolaged boys with fragile $X$ syndrome: Effects of a parentimplemented spoken language intervention. Journal of Communication Disorders, 72, 64-76.

*Puente, A., Alvarado, JM., Jiménez, V., \& Martínez, L. (2017). Reading profiles in adolescents with Fragile $X$ Syndrome and Down syndrome. Anales de psicología, 33(3), 660-669.

Ribate, MP., Pié, J., \& Ramos, FJ. (2010). Síndrome de X Frágil. Protocolo diagnóstico. Asociación Española de Pediatría, 1, 85-90.

*Roberts, J., Mirret, P., Anderson, K., Burchinal, M., \& Neebe, E. (2002). Early communication, symbolic behavior, and social profiles of young males with fragile $X$ syndrome. American Journal of Speech-Language Pathology, 11(3), 295-304.

*Roberts, J., Martin, G., Moskowitz, L, Harris, A., Foreman, J., \& Nelson, L. (2007). Discourse Skills of Boys with Fragile $X$ Syndrome in Comparison to Boys with Down syndrome. Journal of Speech, Language, and Hearing Research, 50, 475-492.

*Robles, MA. (2011). Un caso de Síndrome $X$ frágil y su intervención desde la Atención Infantil Temprana. Electronic Journal of Research in Educational Psychology, 9(3), 13331352. 
*Robles, MA. Sánchez, D. y Valencia, N. (2013). Utilidad de la logopedia en la mejora del lenguaje en el tratamiento del Síndrome del cromosoma $X$ frágil en niños preescolares. Revista de Logopedia, Foniatría y Audiología, 33, 146-154.

*Sterling, A., \& Abbeduto, L. (2012). Language development in school-age females with Fragile $X$ Syndrome. Journal Intellect Disabilities Research, 56(10), 974-983.

Sutherland, GR. (1977). Fragile sites on human chromosomes: demonstration of their dependence on the type of tissue culture medium. Science, 197, 265-266.

Verkerk, AJ, Pieretti, M., Sutcliffe JS, Fu YH, Kuhl DP, Pizzuti, A., ..., \& Eussen, BE (1991). Identification of a gene (FMR-1) containing a CGG repeat coincident with a breakpoint cluster region exhibiting length variation in fragile $X$ syndrome. Cell, 65(5), 905-914.

*Warren, S., Brady, N., Sterling, A., Fleming, K., \& Marquis, J. (2011). Maternal Responsivity Predicts Language Development in Young Children with Fragile $X$ Syndrome. American Journal Intellectual Developmental Disabilities, 115(1), 54-75. 